\title{
Does family background affect job allocation for college graduates in China?
}

\author{
Changjun YUE \\ Peking University, China
}

\begin{abstract}
Based on large-scale national survey data on college graduates employment in China, this paper explores the impact of family background on job allocation, through both descriptive and regression analyses. The result shows as follows. Firstly, family occupational, educational and regional status are significant influential factors. College graduates with better family occupational and educational background, living in big/medium cities have more chances to enter Emerging Industry, Monopoly Industry, Government Industry and SECH Industry. Secondly, College graduates with better family occupational and educational background, living in big/medium cities have more chances to enter Governments Sector and State-Owned Enterprise. Better family educational background may help college graduates enter Foreign-Invested Enterprise. Since the difference between the primary and secondary labor market is very large, the finding that college graduates with better family socio-economic background have more chances to enter primary labor market reveals an unfair competition in China.
\end{abstract}

Key words: higher education; employment allocation; labor market

\section{Introduction}

Since the implementation of the reform and opening door policy in 1978, China has enjoyed a long-term and fast-growing economic boom. China's GDP reached to about 10.4 trillion US dollars in 2014 (National Bureau of Statistics, 2015). Meanwhile, the income gap is so large that the Gini coefficient in 2014 exceeded 0.469. Income gap in China reveals among industries, sectors, cities, regions and so on.

Since 1999, the scale of higher education in China began to expand quickly and the entrance rate of higher education has greatly improved. In 2002, the gross entrance rate of higher education reached $15 \%$, indicating that China entered the era of mass higher education. By 2013, the rate rose to 34.5\% and the gross scale of higher education reached 34.6 million (Ministry of Education, 2014). Meanwhile, the number of college graduates increased gradually, which rises to 7.49 million in 2015. The employment of college graduates has become a key problem faced by Chinese government. College graduate not only feel difficulty to find a job but also feel hard to get a good job which can reach their expectation. Because China's job market is still in transition, the wages in different industries/sectors vary a lot. The theory of labor market segmentation can explain the huge gap between the primary sector and the secondary sector in China. Usually, people working in emerging industry and monopoly industry or in Foreign-invested enterprises and State-owned enterprises can obtain higher wages.

Parents' education, income, or occupations affect children's education and their earnings, and the wage premium of having a cadre parent is $15 \%$ (Li, 2012). Father's education had positive impacts on a Chinese college graduate's educational and employment outcomes (Li, 2010). Wang (2012) found the college premium is larger for people in state-owned enterprise in China. Is job allocation determined by merit? Does family background play an important role? May college graduates from families of higher socioeconomic status have higher earnings than those of lower socioeconomic status? This paper will focus on the job distribution of Chinese college graduates from different family backgrounds. 


\section{Sample description}

\section{Data}

The data set is from National College Graduates Employment (NCGE) survey conducted by Peking University in June, 2013. This survey includes 30 universities/colleges in 21 provinces. There are nine "211-Project" universities which means elite universities in China, nine ordinary universities, seven two-year colleges or vocational colleges, three independent college, and two private universities. The survey records students' demographic characteristics (including family background), scholarly performance, perception and activities with regard to job search, and final outlets including job location and starting salaries. There are 15060 valid sheets turned in. This paper selects a subsample which only includes students with a bachelor degree. After excluding the observations with missing or invalid values of the key variables, our final sample contains 10206 individuals.

\section{Variable description}

Industry: There are 19 kinds of industries in China, this paper merged them into 6 groups. The first group is Emerging Industry including "Information Transmission, Software and Information Technology", "Financial Intermediation" and "Real Estate". The second group is Government Industry including "Public Management, Social Security and Social Organization". The third group is SECH Industry including "Scientific Research and Technical Services", "Education", "Culture, Sports and Entertainment" and "Health and Social Service". The fourth group is Monopoly Industry including "Production and Supply of Electricity, Heat, Gas and Water", which is related to natural resource and owned mainly by state. The fifth group is Manufacturing Industry which belongs to a relative competitive market. The sixth group is Other Industry including 9 remaining industries, namely: "Agriculture, Forestry, Animal, Husbandry and Fishery" ,"Mining”, "Construction”, "Wholesale and Retail Trades", "Transport, Storage and Post”, "Hotels and Catering Services", "Leasing and Business Services", "Management of Water Conservancy, Environment and Public Facilities”, “Services to Households, Repair and Other Services”.

Sector: There are 12 kinds of sectors in the survey. This paper merged them into six groups as follows: Governments, State-Owned Enterprise, SECH Sector (Science, Education, Culture, Health and so on), Foreign-Invested Enterprise, Domestic Private Enterprise, and Other Sector.

Family occupational status: This paper deals family occupational status with parents' occupation as a proxy variable and analyzes its influence on student's employment opportunity. 9 major types of occupations are involved in the survey. According to specific national conditions, this paper defines levels of occupations as follows: (1) government official, organizational staff, manager and specialist, all of which enjoy higher social prestige, as "Upper occupation". (2) Individual businessman, sales, private entrepreneur, laborer, rural migrant worker and farmer, which enjoy lower social prestige, as “Lower occupation”.

Family educational status: This paper deals family educational status with parents' academic qualification as a proxy variable. The types of parents' academic qualifications in the survey are classified into seven groups. This paper defines the former 3 groups (graduate school, 4-year university and 2-year college) as "College 
degree and above", and the latter 4 groups (high-school \& equivalence, junior middle school, primary school, and illiterate or semiliterate) as "High school and below".

Family economic status: Family income per capita is a key variable of the economic family background. It is a categorical variable with seven intervals in the survey. This paper defines family with income per capita less than 10,000 Yuan as "Low income", family with income per capita between 10,001 Yuan and 20,000 Yuan as "Middle income", family with income per capita greater than 20,001 Yuan as "High income".

Family regional status: In the survey home area is classified into five groups. This paper defines metropolis and medium city as "Big/middle city", and county/town, small town and countryside as "County and below".

\section{Statistical description}

Table 1 shows the comparison on monthly starting salary. It shows that college graduates from families of higher socioeconomic status have higher earnings than those of lower socioeconomic status. (1) The monthly starting salary of college graduates from families of upper occupation is $12.0 \%$ higher than that of other college graduates. (2) The monthly starting salary of college graduates from families of college education and above is $13.4 \%$ higher than that of other college graduates. (3) The monthly starting salary of college graduates from families of middle income and high income are $8.8 \%$ and $18.4 \%$ higher than that of college graduates from families of low income, respectively. (4) The monthly starting salary of college graduates from families of big/middle city is $6.2 \%$ higher than that of other college graduates.

Table 1. Statistical description of monthly starting salary

\begin{tabular}{|c|c|c|c|}
\hline Variable & Mean(Yuan) & Std. Dev.(Yuan) & $\mathrm{N}$ \\
\hline \multicolumn{4}{|l|}{ By parents' occupation: } \\
\hline Lower occupation & 3155 & 1281 & 2356 \\
\hline Upper occupation & 3533 & 1655 & 919 \\
\hline \multicolumn{4}{|l|}{ By parents' education level: } \\
\hline High School and below & 3156 & 1279 & 2375 \\
\hline College degree and above & 3578 & 1676 & 849 \\
\hline \multicolumn{4}{|c|}{ By annul family income per capita: } \\
\hline Low income family & 3006 & 1238 & 1129 \\
\hline Middle income family & 3272 & 1294 & 1196 \\
\hline High income family & 3558 & 1667 & 922 \\
\hline \multicolumn{4}{|l|}{ By Home location: } \\
\hline County and below & 3172 & 1283 & 1794 \\
\hline Big/middle city & 3370 & 1530 & 1419 \\
\hline
\end{tabular}


Table 2 shows the comparison on monthly starting salary by employment industry. It shows that there are significant gaps among the incomes of 6 kinds of industries. Emerging Industry enjoys the highest monthly starting salary averaging 3660 Yuan. Monopoly Industry ranks the second highest monthly starting salary averaging 3317 Yuan. Thus, most college graduates regard these two industries as ideal employment goal. Although the monthly starting salaries of both Government Industry and SECH Industry are not high, these two industries in China have more invisible welfare and benefited from stable jobs and better working environment. Therefore, these two industries also receive welcome from college graduates.

Table 2. Monthly starting salary comparison by industry (Yuan)

\begin{tabular}{lcccccc}
\hline \hline Industry & $\begin{array}{c}\text { Emerging } \\
\text { Industry }\end{array}$ & $\begin{array}{c}\text { Government } \\
\text { Industry }\end{array}$ & $\begin{array}{c}\text { SECH } \\
\text { Industry }\end{array}$ & $\begin{array}{c}\text { Monopoly } \\
\text { Industry }\end{array}$ & $\begin{array}{c}\text { Manufacturing } \\
\text { Industry }\end{array}$ & $\begin{array}{c}\text { Other } \\
\text { Industry }\end{array}$ \\
\hline Salary & 3660 & 3132 & 3043 & 3317 & 3129 & 3114 \\
\hline \hline
\end{tabular}

Table 3 shows the comparison on monthly starting salary by employment sector. It shows that there are significant gaps among the incomes of 6 kinds of sectors. Foreign-invested enterprise enjoys the highest monthly starting salary. State-owned enterprise ranks the second place. These two sectors are college graduates' ideal job units. Although the monthly starting salaries of both Government sector and SECH sector are not high, these two sectors can provide more invisible welfare, job stabilization and better environment. Thus, these two sectors are also college graduates' choice.

Table 3. Monthly starting salary comparison by sector (Yuan)

\begin{tabular}{lcccccc}
\hline \hline Sector & Governments & $\begin{array}{c}\text { State-owned } \\
\text { enterprise }\end{array}$ & SECH & $\begin{array}{c}\text { Foreign- } \\
\text { invested } \\
\text { enterprise }\end{array}$ & $\begin{array}{c}\text { Domestic } \\
\text { private } \\
\text { enterprise }\end{array}$ & Sector \\
\hline Salary & 3251 & 3387 & 2909 & 3625 & 3133 & 3207 \\
\hline \hline
\end{tabular}

\section{Regression analysis}

\section{Regression model}

This section analyses the influential factors on college graduates' job allocation using regression model. The dependent variable represents different types of industries/ sectors, so it is a categorical dependent variable, with the value equals to $1,2,3,4,5$ and 6, respectively. Therefore, Multinomial Logit model will be used, with one type as the base category. This paper sets the group of Manufacture Industry / Domestic private enterprise as base category. The regression model of is as follows:

$$
\operatorname{Logit}\left(P_{i / 5}\right)=\ln \left(\frac{P(Y=i \mid X)}{P(Y=5 \mid X)}\right)=\beta_{i 1}+\beta_{i 2} X_{2}+\cdots \beta_{i K} X_{K} \quad(i=1,2,3,4,6)
$$

The independent variables can be divided into three groups. The first group is graduates' family background variables including: (1) Parents' occupation. (2) Parents' schooling year on average. (3) Family economic status, classified into Low income family (accounting for 37.5\%), Middle income family (accounting for 35.4\%), 
and High income family (accounting for 27.1\%), using Low income family as the base category. (4) Home location. The second group is individual personal characteristics, including: (1) Gender. (2) Minority or not. (3) Only one child or not in family. The third group is graduates' human capital variables, including: (1) College's prestige, classified into "211-project" elite university, ordinary four-year university, other university (two-year college or vocational college, independent college and private university), with the last one as the base category. (2) Academic grades, classified into top 25\% group and last $75 \%$ group. (3) English certificate. (4) Communist Party of China (CPC) member.

\section{Regression results}

Table 4 shows the regression results examining the effect of family background on the job opportunity of college graduates to enter each industry. In general, family occupational, educational and regional status are all significant influential factors on chances to enter Emerging Industry, Monopoly Industry, Government Industry and SECH Industry compared to Manufacturing Industry. Graduates with better family occupational and educational background, living in big/medium cities have more chances to enter Emerging Industry, Monopoly Industry, Government Industry and SECH Industry.

(1) Parents' occupation is a significant explanatory variable, as three out of four coefficients of it are positive significantly, indicating that students with superior occupational background have better chance to enter Emerging Industry, Government Industry and SECH Industry. (2) Parents' average schooling year is also a significant explanatory variable, as two out of four coefficients are positive significantly, indicating that students with high academic qualification family have better chance to enter Emerging Industry and Monopoly Industry. (3) Home location a significant explanatory variable as well, since three out of four coefficients are positive and statistically significant, indicating that students living in big/medium cities have better chance to enter Emerging Industry, Monopoly Industry and SECH Industry. (4) Family economic status seems not to be an important variable since most of coefficients are insignificant. The reason may be that the family income per capita is not an ideal variable in that most students don't know the exact number of family income. Besides, human capital and personal characteristics also play important roles on job allocation. Graduates with good academic grades from elite university and from one-child family have better chance to enter Emerging Industry, Monopoly Industry, Government Industry and SECH Industry compared to Manufacturing Industry. 
Table 4. Probability of entering each industry

\begin{tabular}{|c|c|c|c|c|}
\hline Dependent variables & $\begin{array}{c}\text { Emerging } \\
\text { Industry } \\
\text { (1) }\end{array}$ & $\begin{array}{l}\text { Government } \\
\text { Industry } \\
\text { (2) }\end{array}$ & $\begin{array}{c}\text { SECH } \\
\text { Industry } \\
\text { (3) }\end{array}$ & $\begin{array}{c}\text { Monopoly } \\
\text { Industry } \\
\text { (4) }\end{array}$ \\
\hline $\begin{array}{l}\text { Parents’ occupation } \\
\text { (Upper occupation=1) }\end{array}$ & $\begin{array}{l}0.3202^{*} \\
(0.1843)\end{array}$ & $\begin{array}{l}0.6215^{* *} \\
(0.3058)\end{array}$ & $\begin{array}{l}0.5351^{* * *} \\
(0.2066)\end{array}$ & $\begin{array}{c}0.3550 \\
(0.2567)\end{array}$ \\
\hline Parents' schooling year & $\begin{array}{l}0.0590^{* * *} \\
(0.0221)\end{array}$ & $\begin{array}{c}0.0539 \\
(0.0426)\end{array}$ & $\begin{array}{c}0.0220 \\
(0.0252)\end{array}$ & $\begin{array}{l}0.1071^{* * *} \\
(0.0322)\end{array}$ \\
\hline Middle income family & $\begin{array}{c}0.0242 \\
(0.1439)\end{array}$ & $\begin{array}{l}-0.1431 \\
(0.2966)\end{array}$ & $\begin{array}{l}-0.0576 \\
(0.1634)\end{array}$ & $\begin{array}{l}-0.3511^{*} \\
(0.2070)\end{array}$ \\
\hline High income family & $\begin{array}{c}0.2391 \\
(0.1684)\end{array}$ & $\begin{array}{c}0.0724 \\
(0.3113)\end{array}$ & $\begin{array}{l}-0.2205 \\
(0.1957)\end{array}$ & $\begin{array}{l}-0.4766^{*} \\
(0.2545)\end{array}$ \\
\hline $\begin{array}{l}\text { Home location } \\
\text { (Big/middle city=1) }\end{array}$ & $\begin{array}{l}0.3975^{* * *} \\
(0.1337)\end{array}$ & $\begin{array}{c}0.1648 \\
(0.2505)\end{array}$ & $\begin{array}{l}0.3276^{* *} \\
(0.1538)\end{array}$ & $\begin{array}{l}0.4531^{* *} \\
(0.1948)\end{array}$ \\
\hline $\begin{array}{l}\text { Gender } \\
(\text { Male=1) }\end{array}$ & $\begin{array}{l}-0.7084^{* * *} \\
(0.1377)\end{array}$ & $\begin{array}{c}-1.0291^{* * *} \\
(0.2441)\end{array}$ & $\begin{array}{c}-0.9887^{* * *} \\
(0.1553)\end{array}$ & $\begin{array}{l}0.4365^{*} \\
(0.2369)\end{array}$ \\
\hline $\begin{array}{l}\text { Minority } \\
(\text { Han=1) }\end{array}$ & $\begin{array}{l}-0.4749^{*} \\
(0.2524)\end{array}$ & $\begin{array}{l}-0.4769 \\
(0.4394)\end{array}$ & $\begin{array}{l}-0.0296 \\
(0.3015)\end{array}$ & $\begin{array}{l}-0.5144 \\
(0.3346)\end{array}$ \\
\hline $\begin{array}{l}\text { One child } \\
\text { (One child=1) }\end{array}$ & $\begin{array}{l}0.4955^{* * *} \\
(0.1463)\end{array}$ & $\begin{array}{l}1.1473^{* * *} \\
(0.2781)\end{array}$ & $\begin{array}{l}0.5591^{* * *} \\
(0.1671)\end{array}$ & $\begin{array}{l}0.6636^{* * *} \\
(0.2121)\end{array}$ \\
\hline “211-project” elite university & $\begin{array}{l}0.8489^{* * *} \\
(0.1748)\end{array}$ & $\begin{array}{l}1.1064^{* * *} \\
(0.2735)\end{array}$ & $\begin{array}{l}0.8342^{* * *} \\
(0.1934)\end{array}$ & $\begin{array}{l}-0.0179 \\
(0.3029)\end{array}$ \\
\hline Ordinary four-year university & $\begin{array}{c}-0.4487^{* * *} \\
(0.1432)\end{array}$ & $\begin{array}{l}-0.7976^{* *} \\
(0.3516)\end{array}$ & $\begin{array}{l}-0.3926^{* *} \\
(0.1696)\end{array}$ & $\begin{array}{l}0.4647^{* *} \\
(0.1987)\end{array}$ \\
\hline $\begin{array}{l}\text { Academic grades in top 25\% } \\
\text { group (Yes }=1 \text { ) }\end{array}$ & $\begin{array}{l}0.5083^{* * *} \\
(0.1456)\end{array}$ & $\begin{array}{c}0.2418 \\
(0.2672)\end{array}$ & $\begin{array}{l}0.6686^{* * *} \\
(0.1625)\end{array}$ & $\begin{array}{l}0.8928^{* * *} \\
(0.2010)\end{array}$ \\
\hline $\begin{array}{l}\text { English certificate } \\
(\text { Yes }=1)\end{array}$ & $\begin{array}{l}-0.2926^{*} \\
(0.1633)\end{array}$ & $\begin{array}{l}-0.0396 \\
(0.3350)\end{array}$ & $\begin{array}{l}-0.7100^{* * *} \\
(0.1788)\end{array}$ & $\begin{array}{l}-0.6259^{* * *} \\
(0.2170)\end{array}$ \\
\hline $\begin{array}{l}\text { CPC member } \\
(\text { Yes }=1)\end{array}$ & $\begin{array}{c}0.0777 \\
(0.1282)\end{array}$ & $\begin{array}{l}0.8339^{* * *} \\
(0.2417)\end{array}$ & $\begin{array}{c}0.1607 \\
(0.1468)\end{array}$ & $\begin{array}{c}0.0066 \\
(0.1898)\end{array}$ \\
\hline Intercept & $\begin{array}{c}0.4517 \\
(0.3644)\end{array}$ & $\begin{array}{l}-2.3175^{* * *} \\
(0.7037)\end{array}$ & $\begin{array}{c}0.2343 \\
(0.4199)\end{array}$ & $\begin{array}{l}-2.1499^{* * *} \\
(0.5248)\end{array}$ \\
\hline Observations & 3370 & 3370 & 3370 & 3370 \\
\hline Pseudo R-Square & 0.154 & 0.154 & 0.154 & 0.154 \\
\hline
\end{tabular}

Notes: The reference category is Manufacturing Industry. Standard errors in parentheses. ***, ** and * indicate statistical significant levels of $1 \%, 5 \%$ and $10 \%$, respectively.

Table 5 shows the results of logistic regression examining the effect of family background on college graduates' job opportunity enter each sector. In general, family occupational, educational and regional status are all significant influential factors on chances to enter Governments, State-Owned Enterprise and Foreign-Invested 
Enterprise compared to Domestic Private Enterprise. Graduates with better family occupational and educational background, living in big/medium cities have more chances to enter Governments Sector and State-Owned Enterprise. Graduates with better family educational background have more chances to enter Foreign-Invested Enterprise.

Table 5. Probability of entering each sector

\begin{tabular}{|c|c|c|c|c|}
\hline Dependent variables & (1) & $\begin{array}{l}\text { State-owned } \\
\text { enterprises } \\
\text { (2) }\end{array}$ & $\begin{array}{l}\text { SECH } \\
\text { sector } \\
(3)\end{array}$ & $\begin{array}{c}\text { Foreign- } \\
\text { invested } \\
\text { enterprises } \\
\text { (4) }\end{array}$ \\
\hline $\begin{array}{l}\text { Parents' occupation } \\
\text { (Upper occupation=1) }\end{array}$ & $\begin{array}{l}0.3668^{*} \\
(0.2046)\end{array}$ & $\begin{array}{l}0.3166^{* * *} \\
(0.1250)\end{array}$ & $\begin{array}{c}0.2992 \\
(0.1931)\end{array}$ & $\begin{array}{c}0.1318 \\
(0.1903)\end{array}$ \\
\hline Parents' schooling year & $\begin{array}{l}0.1909^{* * *} \\
(0.0303)\end{array}$ & $\begin{array}{l}0.0446^{* * *} \\
(0.0159)\end{array}$ & $\begin{array}{c}0.0310 \\
(0.0252)\end{array}$ & $\begin{array}{l}0.0589^{* *} \\
(0.0254)\end{array}$ \\
\hline Middle income family & $\begin{array}{l}-0.6911^{* * *} \\
(0.2026)\end{array}$ & $\begin{array}{l}-0.2576^{* * *} \\
(0.1056)\end{array}$ & $\begin{array}{l}-0.0978 \\
(0.1667)\end{array}$ & $\begin{array}{l}-0.2017 \\
(0.1707)\end{array}$ \\
\hline High income family & $\begin{array}{l}-0.4247^{* *} \\
(0.2076)\end{array}$ & $\begin{array}{l}-0.1784 \\
(0.1217)\end{array}$ & $\begin{array}{l}-0.3695^{*} \\
(0.1977)\end{array}$ & $\begin{array}{c}0.1342 \\
(0.1833)\end{array}$ \\
\hline $\begin{array}{l}\text { Home location } \\
(\text { Big/middle city=1) }\end{array}$ & $\begin{array}{l}-0.1173 \\
(0.1751)\end{array}$ & $\begin{array}{l}0.2096^{* *} \\
(0.0960)\end{array}$ & $\begin{array}{c}0.1983 \\
(0.1532)\end{array}$ & $\begin{array}{c}0.0481 \\
(0.1492)\end{array}$ \\
\hline $\begin{array}{l}\text { Gender } \\
(\text { Male=1) }\end{array}$ & $\begin{array}{c}0.1553 \\
(0.1687)\end{array}$ & $\begin{array}{l}0.7080^{* * *} \\
(0.0981)\end{array}$ & $\begin{array}{l}-0.1176 \\
(0.1491)\end{array}$ & $\begin{array}{c}0.0021 \\
(0.1445)\end{array}$ \\
\hline $\begin{array}{l}\text { Minority } \\
(\text { Han=1) }\end{array}$ & $\begin{array}{l}-0.4060 \\
(0.3007)\end{array}$ & $\begin{array}{l}-0.3142^{*} \\
(0.1840)\end{array}$ & $\begin{array}{l}-0.3694 \\
(0.2753)\end{array}$ & $\begin{array}{l}-0.0725 \\
(0.3032)\end{array}$ \\
\hline $\begin{array}{l}\text { One child } \\
\text { (One child=1) }\end{array}$ & $\begin{array}{l}0.6844^{* * *} \\
(0.1918)\end{array}$ & $\begin{array}{l}0.4028^{* * *} \\
(0.1038)\end{array}$ & $\begin{array}{c}0.2199 \\
(0.1648)\end{array}$ & $\begin{array}{c}0.0843 \\
(0.1618)\end{array}$ \\
\hline “211-project” elite university & $\begin{array}{c}0.1828 \\
(0.1908)\end{array}$ & $\begin{array}{l}-0.5397^{* * *} \\
(0.1154)\end{array}$ & $\begin{array}{l}-0.2042 \\
(0.1683)\end{array}$ & $\begin{array}{l}-0.0506 \\
(0.1683)\end{array}$ \\
\hline Ordinary four-year university & $\begin{array}{c}0.1270 \\
(0.2050)\end{array}$ & $\begin{array}{c}0.1024 \\
(0.1061)\end{array}$ & $\begin{array}{l}-0.4987^{* * *} \\
(0.1859)\end{array}$ & $\begin{array}{c}0.0897 \\
(0.1707)\end{array}$ \\
\hline $\begin{array}{l}\text { Academic grades in top } 25 \% \text { group } \\
(\text { Yes }=1)\end{array}$ & $\begin{array}{l}-0.0754 \\
(0.1815)\end{array}$ & $\begin{array}{c}0.0954 \\
(0.0995)\end{array}$ & $\begin{array}{c}0.1232 \\
(0.1575)\end{array}$ & $\begin{array}{c}0.0946 \\
(0.1527)\end{array}$ \\
\hline $\begin{array}{l}\text { English certificate } \\
(\text { Yes }=1)\end{array}$ & $\begin{array}{c}0.1163 \\
(0.1953)\end{array}$ & $\begin{array}{l}0.3181^{* * *} \\
(0.1089)\end{array}$ & $\begin{array}{l}-0.2915^{*} \\
(0.1624)\end{array}$ & $\begin{array}{l}0.8308^{* * *} \\
(0.2030)\end{array}$ \\
\hline $\begin{array}{l}\text { CPC member } \\
(\text { Yes }=1)\end{array}$ & $\begin{array}{l}0.5023^{* * *} \\
(0.1643)\end{array}$ & $\begin{array}{l}0.2289^{* * *} \\
(0.0927)\end{array}$ & $\begin{array}{l}-0.0175 \\
(0.1480)\end{array}$ & $\begin{array}{l}-0.0206 \\
(0.1440)\end{array}$ \\
\hline Intercept & $\begin{array}{c}-3.8960^{* * *} \\
(0.4891)\end{array}$ & $\begin{array}{c}-0.9660^{* * *} \\
(0.2625)\end{array}$ & $\begin{array}{c}-1.0270^{* * *} \\
(0.3962)\end{array}$ & $\begin{array}{c}-2.6055^{* * *} \\
(0.4384)\end{array}$ \\
\hline Observations & 3674 & 3674 & 3674 & 3674 \\
\hline Pseudo R-Square & 0.124 & 0.124 & 0.124 & 0.124 \\
\hline
\end{tabular}

Notes: The reference category is Domestic Private Enterprises. Standard errors in parentheses. ***, ** and * indicate statistical significant levels of $1 \%, 5 \%$ and $10 \%$, respectively. 


\section{Conclusions}

Based on national college graduates employment survey data of China, this paper explores the impact of family background on job allocation, through both descriptive and regression analyses. The empirical result shows that the income gap among industries/sectors is significantly large which is in accord with the severe market segmentation (Knight and $\mathrm{Li}, 2005$ ). Emerging Industry, Monopoly Industry, Government Industry and SECH Industry belong to primary labor market in China. Governments Sector, State-Owned Enterprise and Foreign-Invested Enterprise are ideal job sectors for most Chinese college graduates. The empirical result also shows college graduates with better family socio-economic background have more chances to enter primary labor market.

College graduates from elite university are easy to enter the advantage industries/sectors. However, Yue (2015) found that students with better family occupational, educational, regional, and economic status have more chances to enter elite universities. Therefore, employment opportunity inequality in college graduates market has enhanced after China's college expansion. Chinese economy is undergoing middle income trap and job chances become scarce especially for college graduates. How to reduce job inequality in college graduates market is a serious challenge for the effective allocation and use of senior human resources.

\section{References}

Knight, J. and S. Li (2005).Wages, firm profitability and labor market segmentation in urban China. China Economic Review 16, 205-228.

Li, H., L. Meng, X. Shi and B. Wu (2012). Does having a cadre parent pay? Evidence from the first job offers of Chinese college graduates. Journal of Development Economics 99, 513-520.

Li, T. and J. Zhang (2010). What determines employment opportunity for college graduates in China after higher education reform? China Economic Review 21, 38-50.

Wang, L. (2012). Economic transition and college premium in urban China. China Economic Review 23, 238-252.

Yue, C. (2015). Expansion and Equality in Chinese Higher Education. International Journal of Educational Development 40(1), 50-58. 